Perspectives on Maltese Linguistics 


\section{Studia typologica}

Edited by Thomas Stolz, François Jacouesson and Pieter C. Muysken

Beihefte/Supplements Volume 14

STUF - Language Typology and Universals Sprachtypologie und Universalienforschung

Editorial Board

Michael Cysouw (München)

Ray Fabri (Malta)

Steven Roger Fischer (Auckland)

Bernhard Hurch (Graz)

Bernd Kortmann (Freiburg)

Nicole Nau (Poznán)

Ignazio Putzu (Cagliari)

$\dagger$ Anna Siewierska (Lancaster)

Stavros Skopeteas (Bielefeld)

Johan van der Auwera (Antwerpen)

Elisabeth Verhoeven (Berlin)

Ljuba Veselinova (Stockholm) 


\section{Perspectives on Maltese Linguistics}

Albert Borg, Sandro Caruana, Alexandra Vella (Eds.)

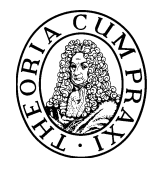

Akademie Verlag 
Bibliografische Information der Deutschen Nationalbibliothek

Die Deutsche Nationalbibliothek verzeichnet diese Publikation in der Deutschen Nationalbibliografie; detaillierte bibliografische Daten sind im Internet über http://dnb.d-nb.de abrufbar.

(C) Akademie Verlag $\mathrm{GmbH}$, Berlin 2014

Ein Wissenschaftsverlag der Oldenbourg Gruppe

www.akademie-verlag.de

Das Werk einschließlich aller Abbildungen ist urheberrechtlich geschützt. Jede Verwertung außerhalb der Grenzen des Urheberrechtsgesetzes ist ohne Zustimmung des Verlages unzulässig und strafbar. Das gilt insbesondere für Vervielfältigungen, Übersetzungen, Mikroverfilmungen und die Einspeicherung und Bearbeitung in elektronischen Systemen.

Redaktion: Cornelia Stroh

Einbandgestaltung: hauser lacour

Druck \& Bindung: Beltz Bad Langensalza GmbH, Bad Langensalza

Dieses Papier ist alterungsbeständig nach DIN/ISO 9706.

ISBN 978-3-05-006275-4

eISBN 978-3-05-006514-4 